\title{
Association between fibroblast growth factor 7 and the risk of chronic obstructive pulmonary disease
}

\author{
Si-cheng $X^{\#}$, Jiang-ying KUANG\#, Jin LIU, Chun-lan MA, Yu-lin FENG, Zhi-guang SU* \\ Molecular Medicine Research Center, West China Hospital, and State Key Laboratory of Biotherapy, Sichuan University, Chengdu \\ 610041, China
}

\begin{abstract}
Aim: Fibroblast growth factor 7 (FGF7) is involved in a number of physiological and pathological processes, including lung disease. However, relatively little is known about the effect of FGF7 gene polymorphisms on chronic obstructive pulmonary disease (COPD) susceptibility. This study aimed to investigate the association between FGF7 polymorphisms with COPD susceptibility in a Chinese Han population.

Methods: We conducted a case-control study of 279 COPD patients and 367 age- and gender-distribution-matched control subjects. The tagging SNPs rs10519225 and rs7170426 in FGF7 were genotyped by SNaPshot. The associations of each SNP genotype and haplotype constructed by these loci with COPD were analyzed.

Results: A multivariate analysis showed that rs10519225 was significantly associated with an increased risk of COPD $(P=0.011$, $\mathrm{OR}=1.535$, FDR $q=0.022$ ), whereas no association was found for rs7170426. Linkage disequilibrium (LD) analysis showed that these loci were in weak LD, with an $r^{2}$ of 0.033 and a D' of 0.232 (95\% Cl: $\left.0.150-0.520\right)$. The haplotype constructed by allele G at rs10519225 and allele A at rs7170426 was associated with a decreased susceptibility to COPD $(P=0.012,0 R=0.751, F D R=0.048)$. Conclusion: These findings suggest that FGF7 may be one susceptibility factor for COPD.
\end{abstract}

Keywords: chronic obstructive pulmonary disease; fibroblast growth factor 7; genetic polymorphism; haplotype

Acta Pharmacologica Sinica (2012) 33: 998-1003; doi: 10.1038/aps.2012.69; published online 16 Jul 2012

\section{Introduction}

Chronic obstructive pulmonary disease (COPD) is currently the leading cause of decreases in disability-adjusted life years (DALY). Globally, it is projected to be the third most important cause of DALY by the year $2020^{[1]}$, and it is estimated that COPD affects nearly $8.2 \%$ of the Chinese adult population ${ }^{[2]}$. Cigarette smoking is the major environmental risk factor for COPD; however, only approximately $15 \%$ of smokers develop clinically relevant airflow obstruction ${ }^{[3]}$. The variation in the susceptibility to cigarette smoke, in combination with the familial inheritance pattern of COPD, suggests that there may be a genetic component to the development of $\mathrm{COPD}^{[4]}$. Multiple studies in diverse populations have demonstrated the genetic contribution to the variability in pulmonary function and the familial aggregation of COPD. As expected, segregation analysis suggests that multiple genes may be involved in COPD susceptibility. The associations between COPD

\footnotetext{
\# These authors contributed equally to this work.

* To whom correspondence should be addressed.

E-mail zhiguang_su@hotmail.com

Received 2011-12-23 Accepted 2012-05-09
}

and polymorphisms in genes with potential importance in COPD pathogenesis have been investigated ${ }^{[5]}$; however, only a1-antitrypsin has been unequivocally identified as relevant to the development of COPD. Recently, polymorphisms in the CHRNA3-CHRNA5-IREB2, HHIP, and FAM13A loci have been found to be associated with COPD by genome-wide association studies (GWAS) ${ }^{[6-8]}$.

Fibroblast growth factor 7 (FGF7), also known as keratinocyte growth factor (KGF), has a variety of effects in the lung. FGF7 stimulates epithelial cell proliferation in the airway and alveoli $^{[9,10]}$ and affects the morphology of the developing lung. The disruption of FGF7 receptor function significantly reduces airway branching during development ${ }^{[11]}$. The over-expression of FGF7 in the airways of developing mice severely alters lung growth ${ }^{[12]}$. FGF7 also stimulates fluid and electrolyte secretion. When applied to fetal lung explants, FGF7 stimulates dramatic intra-luminal swelling and expansion ${ }^{[13]}$. FGF7 may also play a role in airway repair following injury. FGF7 induces alveolar type II pneumocyte proliferation in vitro and in vivo ${ }^{[9]}$. FGF7 stimulates surfactant protein and phospholipid expression as well as the transepithelial transport of fluids and electrolytes, minimizes injury, enhances the repair of dam- 
aged epithelia, and may dampen the epithelial response to inflammatory mediators ${ }^{[14]}$. Exogenous FGF7 treatment has been used as a protective agent after oxidant or bleomycininduced lung injury ${ }^{[15,16]}$. Endogenous FGF7 mRNA expression is induced in neonatal rabbits exposed to hyperoxia, and FGF7 protein expression is increased in adult respiratory distress syndrome ${ }^{[17]}$, suggesting that FGF7 may play a role in lung repair. Thus, this protein could help repair the damaged lungs of individuals who are smokers and who are at a risk of developing COPD.

In addition, FGF7 has been identified to play roles in several clinical diseases or phenotypes, including nonsyndromic cleft lip and palate ${ }^{[18]}$, thyroid volume and goiter risk ${ }^{[19]}$. Moreover, FGF7 was identified as a COPD susceptibility locus in a recent report ${ }^{[20]}$.

With these considerations in mind, we hypothesized that polymorphisms in the FGF7 gene might modulate susceptibility to COPD. To test this hypothesis, we investigated the association between FGF7 gene polymorphisms and the risk of COPD in a Chinese Han population.

\section{Materials and methods \\ Subjects}

As described previously ${ }^{[21]}, 279$ patients with COPD and 367 age-matched non-COPD control subjects were recruited for this study. The subjects in both groups were unrelated ethnic Han Chinese individuals recruited from Chengdu city or surrounding regions in the Sichuan Province of western China. Each subject was personally interviewed face-to-face by trained interviewers who collected the patient's demographic data as well as information related to risk factors such as tobacco smoking. The recruitment and the clinical analyses were conducted at the Department of Respiratory Medicine in West China Hospital of Sichuan University; clinical analyses were performed according to the Global Initiative for Chronic Obstructive Lung Disease (GOLD) criteria ${ }^{[22]}$. COPD patients were enrolled when they suffered from cough, sputum production and dyspnea at least upon exertion and showed chronic irreversible airflow limitation defined by an $\mathrm{FEV}_{1}$ (forced expiratory volume in $1 \mathrm{~s}$ ) to FVC (forced vital capacity) ratio $<70 \%$, and $\mathrm{FEV}_{1}$ predicted $<80 \%$ after the inhalation of a $\beta_{2}$-agonist. Patients were excluded from this study if they had other significant respiratory disease, such as bronchial asthma, bronchiectasis, lung cancer, or pulmonary tuberculosis. The age-matched non-COPD control subjects were volunteers who came to the West China Hospital of Sichuan University for physical examination only. The inclusion criteria for controls were as follows: (1) $\mathrm{FEV}_{1} / \mathrm{FVC}$ ratio $>70 \%, \mathrm{FEV}_{1} \%$ and $\mathrm{FVC} \%$ predicted $>80 \%$ and (2) without pulmonary disease. Individuals were excluded if they had a history of chronic lung disease, atopy, an acute pulmonary infection in the 4 weeks before assessment for this study, or a family history of COPD.

This study was approved by the Ethics Committee of the West China Hospital, Sichuan University, and written informed consent was obtained from all subjects before their participation in the study. The investigator explained the nature, purpose and risks of the study and provided the subject with a copy of the information sheet.

\section{SNP selection}

Based on the linkage disequilibrium and haplotype block analysis of the HapMap project data (http:/ / www.hapmap.org; Public Release \#24/Phase II, Nov 2008), we excluded SNPs with low heterozygosity (below 0.1 ), with low minor allele frequency (below 0.05), or without genotype information. Finally, we chose two TagSNPs at the FGF7 locus, rs10519225 and rs7170426, using the program Tagger with a cut-off of 0.8 for $r^{2}$ and a MAF (minor allele frequency) of $>0.1$. rs10519225 is located at bp 49720778 of chromosome 15 (build 37.3) and captures SNPs, including rs17479589, rs11855798, rs17478785, rs4389093, rs10519225, rs12916839, rs12595692, rs4480740, rs17478694, rs12148764, rs2899434, rs7174135, rs9920722, and rs10519224. Meanwhile, rs7170426 is located at bp 49751853 (build 37.3) and captures SNPs, including rs11630629, rs2899433, and rs7167041. Overall, SNPs rs10519225 and rs7170426 provided $62 \%$ coverage of the genetic information at the FGF7 locus of the Han Chinese population based on MAF $>10 \%$ and $r^{2}>0.8$.

\section{SNP genotyping}

Venous blood was collected from each subject in sterile tubes with EDTA-Na $\mathrm{Na}_{2}$ anticoagulants and stored at $-20^{\circ} \mathrm{C}$. Genomic DNA was extracted from the stored blood using a commercial extraction kit (Bioteke Corporation, Beijing, China) according to the manufacturer's instructions. SNPs were genotyped using the ABI SNaPshot method (Applied Biosystems, CA, USA). PCR was performed using the specific primers: rs10519225 forward 5'-GAAGAAGAGCATTGAGAAC-3', rs10519225 reverse 5'-TCTGTCTTCCACATCTGTC-3', rs7170426 forward 5'-GAACCTGCATTTCAGCATTG-3', and rs7170426 reverse 5'-CTACAAGTCTCAAGCACAGC-3'. The specific $\mathrm{SNaPshot}$ primers were used for a $\mathrm{SNaPshot}$ reaction using the purified PCR products as templates. The following primers were used for the SNaPshot reaction: rs10519225: 5' - ATTCTGCACCTGACAGAAATAACACTGCTCTTTAAGCTGA-3' and rs7170426: 5'-TTTATGTAAATTTATAGAGTTTGAGGAATAATTAAAACTAGTATTTCT-3'. The SNaPshot reaction products were then analyzed on an ABI 3130 Genetic Analyzer (Applied Biosystems, CA, USA). To confirm the genotyping results, $10 \%$ of the samples were randomly selected and re-genotyped by direct sequencing using a BigDye terminator (Applied Biosystems, CA, USA); no more than a $2 \%$ discrepancy was observed for any SNP between the two genotyping methods.

\section{Statistical analysis}

The demographic and clinical data between the COPD patients and the control subjects were compared using the chi-squared test and Student's t-test. Hardy-Weinberg equilibrium was tested with a goodness of fit chi-squared test (with one degree of freedom) to compare the observed and expected genotype frequencies. The differences between the COPD patients and 
the controls with respect to the genotype distributions were analyzed using one-way analysis of variance for univariate analysis and logistic regression for multivariate analysis. Age and sex were used as covariates in the multivariable analyses. A two-sided significance level of $q<0.05$ was used for all significant tests. Statistical analyses were performed in SPSS version 17.0 and Microsoft Excel. To correct for testing for multiple dependent parameters, the false discovery rate (FDR) was calculated using the Benjamini-Hochberg procedure ${ }^{[23]}$. The FDR significance level was set at $q<0.05$. We used SVS7 (SNP \& Variation Suite 7, available at http:/ / www.goldenhelix.com/SNP_Variation/index.html) to calculate the linkage disequilibrium (LD) $\left(r^{2}\right)$ and performed the haplotype association.

\section{Results}

\section{General characteristics}

The baseline characteristics and the results of the pulmonary function tests for the 279 patients with COPD and 367 control subjects were presented in Table 1. All patients had $\mathrm{FEV}_{1}$ values $<80 \%$ of predicted and thus were diagnosed with moderate-to-severe COPD according to the Global Initiative for Chronic Obstructive Lung Disease (classification of severity: mild $=\mathrm{FEV}_{1} \geq 80 \%$ of predicted; moderate $=\mathrm{FEV}_{1} \geq 30 \%$ to $<80 \%$ of predicted; and severe $=\mathrm{FEV}_{1}<30 \%$ of predicted). The COPD cases and control subjects did not significantly differ in sex, age or smoking history. The $\mathrm{FEV}_{1}, \mathrm{FEV}_{1} /$ predicted and $\mathrm{FEV}_{1} /$ FVC were significantly lower in the COPD case subjects compared with the controls $(P<0.01)$.

\section{rs10519225 is associated with COPD}

All subjects were genotyped at both rs10519225 and rs7170426 using the $\mathrm{SNaPshot}$ method. The genotype distributions are indicated in Table 2. The distributions of each genotype of rs10519225 and rs7170426 in COPD patients and controls were compatible with the Hardy-Weinberg equilibrium $\left(x^{2}=1.752\right.$
Table 1. Description of study population.

\begin{tabular}{|c|c|c|c|}
\hline Variable & $\begin{array}{l}\text { Controls } \\
(n=367)\end{array}$ & $\begin{array}{c}\text { Cases } \\
(n=279)\end{array}$ & $P$ \\
\hline Age, years & $65 \pm 8$ & $63 \pm 9$ & NS \\
\hline Sex (Male/Female) & $323 / 44$ & $239 / 40$ & NS \\
\hline \multicolumn{4}{|l|}{ Smoking history } \\
\hline $0-20$ pack years & 88 & 75 & NS \\
\hline$\geq 20$ pack years & 279 & 204 & NS \\
\hline $\mathrm{FEV}_{1}$ & $1.87 \pm 0.60$ & $0.97 \pm 0.32$ & $<0.01$ \\
\hline $\mathrm{FEV}_{1}$ percentage of predicted, $\%$ & $93.7 \pm 3.4$ & $46.0 \pm 0.4$ & $<0.01$ \\
\hline $\mathrm{FEV}_{1} / \mathrm{FVC}, \%$ & $78.0 \pm 4.6$ & $49.2 \pm 8.3$ & $<0.01$ \\
\hline
\end{tabular}

$\mathrm{FEV}_{1}=$ Forced Expiratory Volume in $1 \mathrm{~s}, \quad \mathrm{FVC}=$ Forced Vital Capacity. NS: not significant.

Data are presented as mean \pm SEM.

and 0.347 for COPD patients, respectively; $\chi^{2}=0.652$ and 0.347 for controls, respectively; all $P$ values were higher than 0.05). For rs10519225, the frequencies of the GG, GA, and AA genotypes were $63.8 \%, 33.7 \%$, and $2.5 \%$ in COPD patients and $70.8 \%, 26.2 \%$, and $3.0 \%$ in controls, respectively. For rs7170426, the observed frequencies of the AA, AG, and GG genotypes were $51.6 \%, 39.1 \%$, and $9.3 \%$ in COPD patients and $55.9 \%, 37.6 \%$, and $6.5 \%$ in controls, respectively. In the univariate analysis, the genotypes of rs10519225 were distributed dramatically differently between COPD patients and healthy controls $(P=0.022)$, whereas the distribution of rs7170426 was not different between two groups. Genotypes containing the minor rs10519225 A allele were associated with COPD susceptibility in an additive pattern (Table 2). After adjustment for age and sex, the association of rs10519225 and COPD remained significant $(P=0.011, \mathrm{FDR} q=0.022)$. Multivariate binary logistic regression to estimate the magnitude of effect showed that the odds ratio (OR) for the presence of at least one minor allele

Table 2. Associations of genotypes in rs10519225 and rs 7170426 with COPD.

\begin{tabular}{|c|c|c|c|c|c|}
\hline \multirow[t]{2}{*}{ Genotype } & \multicolumn{2}{|c|}{ Frequency } & \multirow{2}{*}{ Univariate } & \multirow{2}{*}{$\begin{array}{l}\text { Multivariate } P \text { value } \\
\quad\left(\text { FDR } q \text { value }{ }^{\dagger}\right)\end{array}$} & \multirow{2}{*}{ OR $(95 \% \mathrm{Cl})^{\S}$} \\
\hline & COPD & Controls & & & \\
\hline \multicolumn{6}{|c|}{ rs10519225 G/A } \\
\hline GG & 0.638 & 0.708 & 0.023 & $0.011(0.022)$ & $1.535(1.104-2.134)$ \\
\hline GA & 0.337 & 0.262 & & & \\
\hline AA & 0.025 & 0.030 & & & \\
\hline \multicolumn{6}{|c|}{ rs7170426 A/G } \\
\hline AA & 0.516 & 0.559 & 0.135 & $0.079(0.079)$ & $1.324(0.968-1.810)$ \\
\hline$A G$ & 0.391 & 0.376 & & & \\
\hline GG & 0.093 & 0.065 & & & \\
\hline Age & & & 0.931 & 0.933 (0.931) & $0.998(0.945-1.053)$ \\
\hline Sex & & & 0.379 & $0.419(0.762)$ & $1.212(0.760-1.932)$ \\
\hline
\end{tabular}

\footnotetext{
*Binary logistic regression.

${ }^{\dagger}$ FDR (false discovery rate) evaluated for association with SNP only. $q<0.05$ is significant.

${ }^{\S}$ Both rs10519225 and rs 7170426 entered using additive model.
} 
of rs10519225 (additive model) was 1.535 (95\% CI: 1.104-2.134) (Table 2).

\section{Association between haplotype of rs10519225 and rs7170426 with COPD}

LD analysis showed that rs10519225 and rs7170426 were in weak LD, with $r^{2}=0.033, D^{\prime}=0.232$, and $D^{\prime} 95 \%$ CI (0.150-0.520). Multivariate logistic regression was used to incorporate age, sex, and different rs10519225 and rs7170426 haplotypes into the models. As shown in Table 3, the G-A haplotype of rs10519225-rs7170426 was associated with a lower risk of COPD under the additive model (OR=0.751, 95\% CI: 0.598 $0.944, P=0.012$, FDR $q=0.048$ ), whereas none of the other haplotypes were found to have significant effects.

Table 3. Associations of haplotypes between rs10519225 and rs7170426 with COPD*.

\begin{tabular}{lccccc}
\hline $\begin{array}{l}\text { Haplo- } \\
\text { type }\end{array}$ & CASE & Controls & OR $(95 \% \mathrm{Cl})$ & $P$ value $^{\dagger}$ & FDR q $^{\S}$ \\
\hline rs10519225-rs7170426 & & & \\
G-A & 0.594 & 0.660 & $0.751(0.598-0.944)$ & 0.012 & 0.048 \\
G-G & 0.200 & 0.179 & $1.149(0.868-1.521)$ & 0.318 & 0.318 \\
A-A & 0.111 & 0.094 & $1.193(0.831-1.714)$ & 0.304 & 0.405 \\
A-G & 0.095 & 0.066 & $1.485(0.990-2.227)$ & 0.032 & 0.065 \\
\hline
\end{tabular}

* The association analyses were performed with sex, and age as covariates.

${ }^{\dagger}$ Significant $P$ values are shown in boldface.

${ }^{\S}$ FDR, false discovery rate, $q<0.05$ is significant.

\section{Discussion}

Case-control association studies of a candidate gene can be a very powerful approach toward identifying the genetic causes of complex diseases such as COPD ${ }^{[24]}$. In this case-control study, we evaluated the possible association of FGF7 gene polymorphisms with susceptibility to COPD in a Chinese Han population for the first time. Our results suggested that the rs10519225 polymorphism in the FGF7 gene correlated with modestly higher risks of COPD, with an OR of 1.54 (95\% CI: 1.104-2.134), whereas the rs7170426 polymorphism was not correlated with COPD risk. Haplotype analysis, in which several SNPs within the same gene are evaluated simultaneously, can provide more information than a single SNP and thus elevates the statistical power of the analysis ${ }^{[25]}$. LD analysis showed that these loci were in weak LD. Therefore, we assayed each of the haplotypes constructed by polymorphisms rs10519225 and rs7170426. The G-A haplotype was associated with a lower risk of COPD in the additive genetic model (OR=0.751, 95\% CI: 0.598-0.944, $P=0.012$, FDR $q=0.048)$. This suggested that the G-A haplotype might serve as a protective genetic factor against COPD in Chinese Han population. Nonetheless, further studies are required to confirm this hypothesis. These results indicated that certain polymorphisms in the FGF7 gene may be crucial in the pathogenesis of COPD. Intriguingly, the human FGF7 gene is located on chromosome 15 at position q21.2, and a previous genomewide linkage study for generalized COPD identified chromosome $15 q 21$ as meeting genome-wide criteria for suggestive linkage ${ }^{[26]}$. Moreover, another polymorphism within the FGF7 gene, rs4480740, was recently reported to be associated with COPD in an additional population ${ }^{[20]}$. We assessed the extent of LD between rs10519225, rs7170426, and rs4480740 in the Han Chinese population using HapMap project data. Both rs10519225 and rs7170426 were found to be in strong LD with rs4480740 (Figure 1). Although we did not carry out this association study in an independent population, the strong LD between rs10519225 and rs4480740 suggested that the association of rs10519255 with COPD observed in the current study was unlikely to have occurred by chance.

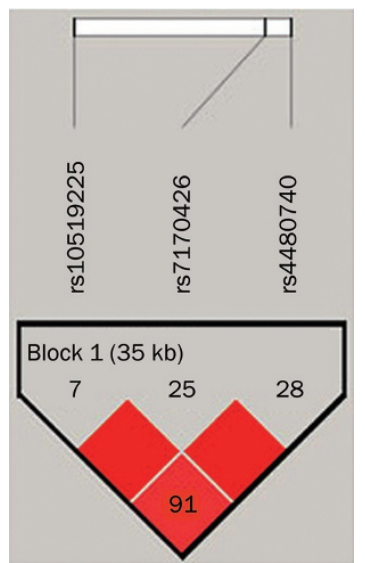

Figure 1. Relative position of SNPs and linkage disequilibrium map for FGF7 in the Han Chinese population populations studied. Polymorphisms are identified by their dbSNP rs numbers, and their relative positions are marked by vertical lines within the white horizontal bar. The numbers within squares indicate the $D^{\prime}$ value, expressed as a percentile.

It should be acknowledged that the SNPs associated with the statistical signal might simply play a role as a surrogate marker for the causal functional SNP or SNPs. That is, rs10519225 in FGF7 gene could be in linkage disequilibrium with another polymorphism of the gene that impacts FGF7 activity. Moreover, rs10519225 is located within an intronic region of FGF7, which is usually removed during the genesplicing process. Intronic SNPs may modify gene function by affecting the regulation of gene expression ${ }^{[27]}$. The dysfunction of FGF7 may pose a risk for the improper development of the airway and alveoli. It has been proposed that persons with smaller initial lung volumes are more likely to develop overt manifestations of disease during the accelerated decline of lung function in COPD. Alternatively, FGF7 may play a role in disease susceptibility through its role in the protection against the oxidative stress response specifically in the lung 
epithelium.

The direction of association with disease is noteworthy; rs10519225 heterozygosity was associated with susceptibility to COPD in this study. The finding of heterozygote susceptibility is unusual in genetic disease association studies, but is well described in the study of genetic susceptibility to human pulmonary or airway diseases, including COPD and asthma $^{[28-30]}$.

We are aware that the significant results in this study could prove to be false positives because of the relatively small sample size, but even with a larger sample, the functional and biological impacts of the described polymorphisms would require further study. Possible gene-gene and gene-environment interactions also pose a challenge for genetic analysis of COPD association studies. Further studies using larger populations are needed, and other variants in the FGF7 gene should be investigated to clarify the association of FGF7 and individual susceptibility to the development of COPD.

In conclusion, we have investigated the link between FGF7 gene polymorphisms and the risk of COPD in the Chinese Han population for the first time. The rs10519225 genotype frequencies are significantly different in COPD patients and controls, suggesting that this SNP may be useful for predicting COPD susceptibility. Similarly, the rs10519225G-rs7170426A haplotype may be protective against COPD in the Chinese Han population. Further functional characterization of these SNPs is required to clarify the significance of FGF7 in the pathogenesis of COPD.

\section{Acknowledgements}

This study was financially supported by the National Natural Science Foundation of China (№ 31071108), the Program for New Century Excellent Talents in University (NCET-10-0600), the Scientific Research Foundation for Returning Overseas Chinese Scholars, the Chinese Ministry of Education (2011508-4-3), and the General Administration of Quality Supervision, Inspection and Quarantine (GAQSIQ) Public Benefit Research Foundation (№ 201210046).

\section{Author contribution}

Si-cheng XU, Jiang-ying KUANG, Jin LIU, Chun-lan MA, and Yu-lin FENG contributed to the study design and genotyping; Zhi-guang SU contributed to the study design and data analysis and wrote the manuscript.

\section{References}

1 Murray CJ, Lopez AD. Evidence-based health policy - lessons from the Global Burden of Disease Study. Science 1996; 274: 740-3.

2 Zhong N, Wang C, Yao W, Chen P, Kang J, Huang S, et al. Prevalence of chronic obstructive pulmonary disease in China: a large, populationbased survey. Am J Respir Crit Care Med 2007; 176: 753-60.

3 Davis RM, Novotny TE. The epidemiology of cigarette smoking and its impact on chronic obstructive pulmonary disease. Am Rev Respir Dis 1989; 140: S82-4.

4 Khoury MJ, Beaty TH, Tockman MS, Self SG, Cohen BH. Familial aggregation in chronic obstructive pulmonary disease: use of the loglinear model to analyze intermediate environmental and genetic risk factors. Genet Epidemiol 1985; 2: 155-66.

5 Castaldi PJ, Cho MH, Cohn M, Langerman F, Moran S, Tarragona N, et al. The COPD genetic association compendium: a comprehensive online database of COPD genetic associations. Hum Mol Genet 2010; 19: 526-34.

6 Cho MH, Boutaoui N, Klanderman BJ, Sylvia JS, Ziniti JP, Hersh CP, et al. Variants in FAM13A are associated with chronic obstructive pulmonary disease. Nat Genet 2010; 43: 200-2.

7 Pillai SG, Ge D, Zhu G, Kong X, Shianna KV, Need AC, et al. A genomewide association study in chronic obstructive pulmonary disease (COPD): identification of two major susceptibility loci. PLoS Genet 2009; 5: e1000421.

8 Wilk JB, Chen TH, Gottlieb DJ, Walter RE, Nagle MW, Brandler BJ, et al. A genome-wide association study of pulmonary function measures in the Framingham Heart Study. PLoS Genet 2009; 5: e1000429.

9 Ulich TR, Yi ES, Longmuir K, Yin S, Biltz R, Morris CF, et al. Keratinocyte growth factor is a growth factor for type II pneumocytes in vivo. J Clin Invest 1994; 93: 1298-306.

10 Yano T, Mason RJ, Pan T, Deterding RR, Nielsen LD, Shannon JM. KGF regulates pulmonary epithelial proliferation and surfactant protein gene expression in adult rat lung. Am J Physiol Lung Cell Mol Physiol 2000; 279: L1146-58.

11 Peters K, Werner S, Liao X, Wert S, Whitsett J, Williams L. Targeted expression of a dominant negative FGF receptor blocks branching morphogenesis and epithelial differentiation of the mouse lung. EMBO J 1994; 13: 3296-301.

12 Simonet WS, DeRose ML, Bucay N, Nguyen HQ, Wert SE, Zhou L, et al. Pulmonary malformation in transgenic mice expressing human keratinocyte growth factor in the lung. Proc Natl Acad Sci U S A 1995; 92: 12461-5.

13 Cardoso WV, Itoh A, Nogawa H, Mason I, Brody JS. FGF-1 and FGF7 induce distinct patterns of growth and differentiation in embryonic lung epithelium. Dev Dyn 1997; 208: 398-405.

14 Prince LS, Karp PH, Moninger TO, Welsh MJ. KGF alters gene expression in human airway epithelia: potential regulation of the inflammatory response. Physiol Genomics 2001; 6: 81-9.

15 Baba Y, Yazawa T, Kanegae Y, Sakamoto S, Saito I, Morimura N, et al. Keratinocyte growth factor gene transduction ameliorates acute lung injury and mortality in mice. Hum Gene Ther 2007; 18: 130-41.

16 Sakamoto S, Yazawa T, Baba Y, Sato H, Kanegae Y, Hirai T, et al. Keratinocyte growth factor gene transduction ameliorates pulmonary fibrosis induced by bleomycin in mice. Am J Respir Cell Mol Biol 2011; 45: 489-97.

17 Ware LB, Matthay MA. Keratinocyte and hepatocyte growth factors in the lung: roles in lung development, inflammation, and repair. Am J Physiol Lung Cell Mol Physiol 2002; 282: L924-40.

18 Riley BM, Mansilla MA, Ma J, Daack-Hirsch S, Maher BS, Raffensperger LM, et al. Impaired FGF signaling contributes to cleft lip and palate. Proc Natl Acad Sci U S A 2007; 104: 4512-7.

19 Teumer A, Rawal R, Homuth G, Ernst F, Heier M, Evert M, et al. Genome-wide association study identifies four genetic loci associated with thyroid volume and goiter risk. Am J Hum Genet 2011; 88: 66473.

20 Brehm JM, Hagiwara K, Tesfaigzi Y, Bruse S, Mariani TJ, Bhattacharya S, et al. Identification of FGF7 as a novel susceptibility locus for chronic obstructive pulmonary disease. Thorax 2011; 66: 1085-90.

21 Su ZG, Wen FQ, Feng YL, Xiao M, Wu XL. Transforming growth factorbeta1 gene polymorphisms associated with chronic obstructive pulmonary disease in Chinese population. Acta Pharmacol Sin 2005; 26: 714-20.

22 Fabbri LM, Hurd SS. Global strategy for the diagnosis, management 
and prevention of COPD: 2003 update. Eur Respir J 2003; 22: 1-2.

23 Benjamini Y, Hochberg Y. Controlling the false discovery rate: a practical and powerful approach to multiple testing. J R Stat Soc Series B Stat Methodol 1995; 57: 289-300.

24 Silverman EK, Palmer LJ. Case-control association studies for the genetics of complex respiratory diseases. Am J Respir Cell Mol Biol 2000; 22: 645-8.

25 Morris RW, Kaplan NL. On the advantage of haplotype analysis in the presence of multiple disease susceptibility alleles. Genet Epidemiol 2002; 23: 221-33.

26 Silverman EK, Palmer LJ, Mosley JD, Barth M, Senter JM, Brown A, et al. Genomewide linkage analysis of quantitative spirometric phenotypes in severe early-onset chronic obstructive pulmonary disease. Am J Hum Genet 2002; 70: 1229-39.

27 Korb M, Ke YB, Johnson LF. Stimulation of gene expression by introns: conversion of an inhibitory intron to a stimulatory intron by alteration of the splice donor sequence. Nucl Acids Res 1993; 21: 5901-8.

28 Gaspar P, Moreira J, Kvitko1 K, Torres M, Moreira A, Weimer T. CYP1A1, CYP2E1, GSTM1, GSTT1, GSTP1, and TP53 polymorphisms: do they indicate susceptibility to chronic obstructive pulmonary disease and non-small-cell lung cancer? Genet Mol Biol 2004; 27 : 133-8.

29 Dahl M, Tybjaerg-Hansen A, Lange P, Nordestgaard BG. DeltaF508 heterozygosity in cystic fibrosis and susceptibility to asthma. Lancet 1998; 351: 1911-3.

30 Seersholm N, Wilcke JT, Kok-Jensen A, Dirksen A. Risk of hospital admission for obstructive pulmonary disease in alpha(1)-antitrypsin heterozygotes of phenotype PiMZ. Am J Respir Crit Care Med 2000; 161: 81-4. 